\section{Transabdominal Cerclage Placement in a Gravid Patient at Week 22 of Gestation Period: A Case Report and Review of the Literature}

\section{Abstract}

Cervical insufficiency $[\mathrm{Cl}]$ describes the painless dilation and shortening of the cervix at the end of the first trimester or early in the second trimester of the pregnancy and may result to miscarriage or preterm labor. The usual treatment is transvaginal cerclage [TVC]. There are however patients which should not be submitted in TVC, since TVC cannot be technically performed or may not be effective. In these women, transabdominal cerclage [TAC] placement may be the optimal alternative. We present a case of a gravid patient at week 22 of gestation, who underwent an emergency TAC, due to $\mathrm{CI}$. TAC took place two days after the diagnosis of $\mathrm{Cl}$ successfully, without any complication. The pregnancy proceeded without any other complication and a healthy female infant was delivered in $34^{\text {th }}$ week due to beginning of labor, by elective cesarean section. According to our knowledge, there are no other reports of TAC placement, in a pregnant patient at week 22; a short review of the literature is also presented.

Keywords: Cervical insufficiency; Cerclage; Transabdominal

\section{Fotopoulos $\mathbf{S}^{1}$ Nikolaidou ME${ }^{1}$, Mitsis T¹, Bourgioti $\mathrm{C}^{2}$, Moustakarias $\mathrm{T}^{1}$ and Moulopoulos LA²}

\section{1 laso Maternity Hospital, 37-39 Kifissias Avenue, Athens, Greece 15123 \\ 2 Department of Radiology, School of Medicine, University of Athens, Aretaieion Hospital, Athens, Greece}

*Corresponding author: Nikolaidou ME

” marilianikolaidou@gmail.com

Nikolaidou ME, Department of Gynaecology and Obstetrics, IASO Maternity Hospital, 383, Athens, Greece.

Tel: 00306945977744

Citation: Fotopoulos S, Nikolaidou ME, Mitsis T, Bourgioti C, Moustakarias T, et al. (2020) Transabdominal Cerclage Placement in a Gravid Patient at Week 22 of Gestation Period: A Case Report and Review of the Literature. Gynecol Obstet Case Rep Vol.6 No.2:10

\section{Introduction}

The term cervical insufficiency $[\mathrm{Cl}]$ includes the painless dilation and shortening of the cervix at the end of the first trimester or early in the second trimester of the pregnancy. Despite the absence of uterine activity, $\mathrm{Cl}$ may result to miscarriage or preterm labor [1]. The incidence of $\mathrm{Cl}$ is estimated less than $1 \%$ of all pregnancies [2]; however, it seems to be responsible for about $8 \%$ of the second trimester miscarriages [3] and is, therefore, considered as one of the leading causes of preterm labor and neonatal morbidity and mortality [4].

Cervical insufficiency is usually treated with transvaginal cerclage [TVC] placement, especially when the cervical length is less than $15 \mathrm{~mm}$ or when there is a history of miscarriage, preterm labor or prior surgical procedures at the cervix such as trachelectomy or conization. However, there is a number of cases in which TVC cannot be technically performed or may not be effective [5]. In these women, transabdominal cerclage [TAC] placement may be the optimal alternative.

Initially, eligibility criteria for the transabdominal placement of a cervico-isthmic cerclage in pregnant women with $\mathrm{Cl}$ included only the presence of an extremely short cervix, due to which TVC was technically impossible [6]. Short cervix could be either congenital, associated with connective tissue disorders [e.g. Ehlers-Danlos syndrome] [2,7], which result in insufficient collagen formation or related to prior surgical interventions [e.g. conization, or therapeutic abortions] [2,7]. Currently, there are even more indications for performing TAC, including a previous non-successful TVC placement and history of radical trachelectomy in women treated for early cervical cancer [8].

We present a case of a gravid patient at week 22 of gestation, who underwent an emergency TAC, due to $\mathrm{Cl}$. According to our knowledge, there are no other reports of TAC placement, in a pregnant patient at week 22; a short review of the literature is also presented.

\section{Case Report}

A 46-year-old woman, at week 22 of gestation, was referred to our Unit due to $\mathrm{Cl}$. The patient was nulliparous and had achieved 
a twin pregnancy following an IVF treatment. She had history of myomectomy and a diagnostic hysteroscopy prior to IVF treatment, but no history of any cervical procedure.

During the first trimester of pregnancy, one of the fetuses had a spontaneous miscarriage and the pregnancy continued as singleton pregnancy. Second trimester ultrasound, which was performed at $22+0$ week of gestation showed cervical funneling and shortening [13 $\mathrm{mm}$ ] (Figure 1). The patient was referred to our Hospital for consultation, one day after the US examination. Interestingly, vaginal examination revealed complete dilation of the cervix and prolapse of the membranes. Since the patient had no signs of chorioamnionitis or uterine contractions, she was considered eligible for cerclage placement [9-15].

Taking into consideration all clinical data, the risk of maternal and fetal morbidity, the advanced maternal age and the desire of the patient to retain the pregnancy, an emergency TAC placement was considered the appropriate treatment. Both parents were counseled in detail for possible risks and written informed consent was obtained from the mother.

TAC placement was successfully performed, two days after the diagnosis of $\mathrm{Cl}$. The operation was performed under general anesthesia, operating time was 45 minutes and the estimated blood loss was less than $50 \mathrm{ml}$. The abdominal cavity was entered through a Pfannesteil incision; first, the vesicouterine peritoneal fold was revealed and dissected, and then, the bladder was mobilized inferiorly by sharp dissection and the uterus was carefully and gently elevated out of the abdominal cavity. This manoevre allowed the surgeon to identify the uterine vessels as well as the isthmus and the uterosacral ligaments at the posterior surface of the uterus. The cerclage [Mersilen-5mm] was inserted in the avascular area between the ascending and descending branches of the uterine artery, above the uterosacral ligaments and, via the avascular area, it was positioned to the other side of the uterus. The band was tied on the anterior surface of the uterus. After ensuring hemostasis and checking both parametrial spaces for potential bleeding, the uterus was placed back into the pelvic cavity and the visceral peritoneum was sutured above the knot.

There were no perioperative complications; the patient had an uneventful post-op course and was finally discharged on the 6th day after surgery. Repeat US examination, which was performed one day after the procedure, showed a cervical length of $21 \mathrm{~mm}$ (Figure 2).

The pregnancy proceeded uneventfully until the $34^{\text {th }}$ week. Due to uterine contraction and the beginning of labor, an elective cesarean section was performed; the cerclage suture was removed during the procedure. A healthy female infant weighing 1760 gram was delivered, with Apgar score $9 / 10$ in $1^{\text {st }}$ and $5^{\text {th }}$ minute. The mother was discharged at the fourth day after the delivery; no maternal complications were reported during or after the delivery. The infant was admitted to the neonatal unit and was discharged twenty seven days after delivery, weighting 2310 gram without any health problems.

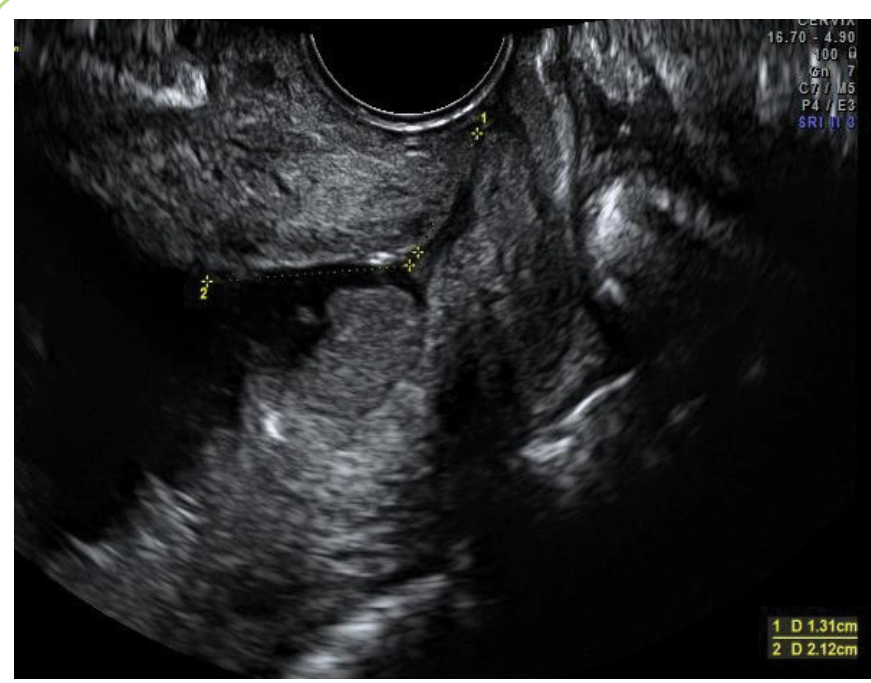

Figure 1 A 42-year-old gravid patient at week 22 of gestation. Sagittal TVUS image demonstrates complete cervical dilatation, before TAC placement. [TVUS: Transvaginal Ultrasound].

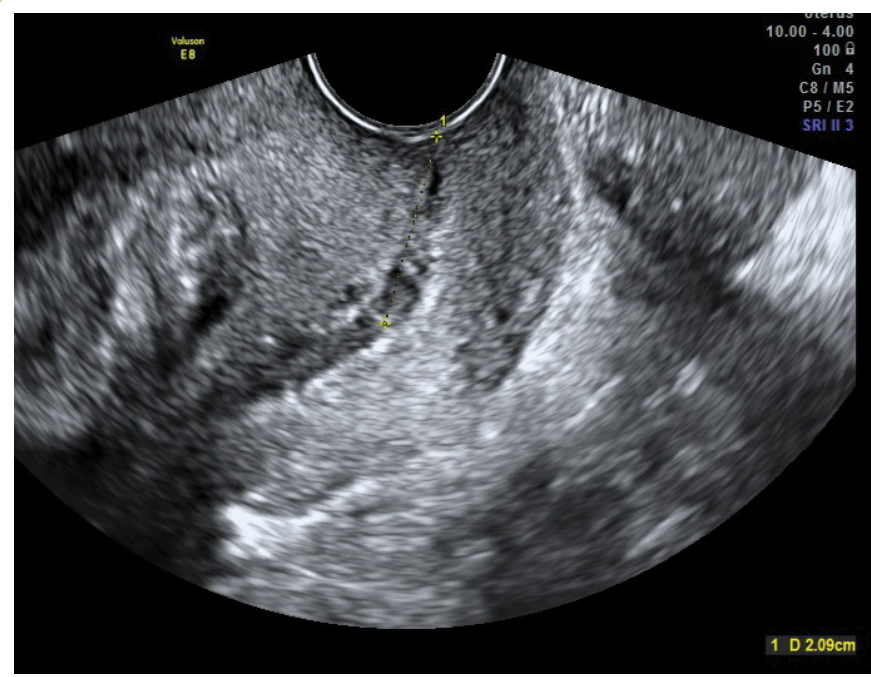

Figure 2 Corresponding sagittal TVUS image of the same patient as in Figure 1 demonstrates cervical length after the TAC procedure.

\section{Discussion}

We report a case of a gravid patient at week 22 of gestation who underwent an emergency TAC placement due to complete cervical incompetence; despite the advanced gestational age, TAC positioning was successfully performed, without maternal or fetal complications, prolonging the course of pregnancy to the $34^{\text {th }}$ week.

Cerclage placement may be characterized as emergency or prophylactic, according to the status of the cervix; an emergency cerclage placement is considered when there is dilation of the cervix $>1-2 \mathrm{~cm}$ [clinically or sonographically confirmed] without uterine contractions and with or without membrane 
bulging through the external cervical os [2]; prophylactic cerclage placement is considered in cases of adverse obstetrical history of the mother [previous premature labor, conization, etc.]. Emergency cerclage placement seems to be effective in prolonging pregnancy. In a small randomized trial, prolongation time was up to 4 weeks [16], while in other observational studies it ranged between 6 and 9 weeks, compared to that of conservative treatment [bed rest] [16-19]. However, these studies included mostly TVC cases.

TVC technique was first introduced by Shirodkar [9] and McDonald [10] in 1950, while the TAC route was described ten years later [6]. Differences between the two techniques included the site of cerclage suture placement, since in TAC the suture is placed at the level of the cervico-isthmic junction, while in TVC, it is placed at the junction of the cervix and vaginal fornix [8]. This results in a different mechanism of action between the two types of cerclage, and it seems that TAC offers higher structural support. TAC is not routinely performed, as it is an extremely difficult procedure from a technical point of view and it is associated with higher morbidity rates than TVC [11]; however, it may be the only option in a small number of patients in whom TVC cannot be performed. TVC is contraindicated in the presence of an extremely short cervix [congenital or acquired], in cases of distorted cervical anatomy [8] including an amputated cervix due to trachelectomy or conization, a scarred cervix due to previous unsuccessful TVC or previous obstetrical trauma [4] and in cases of advanced [second trimester] gestational age. In our case, TAC was the only feasible solution, because of the complete cervical dilation and the advanced gestational age, which rendered TVC impossible.

Laparoscopy TAC was introduced to clinical practice during the last decade. However, this procedure is feasible until the $16^{\text {th }}$ $17^{\text {th }}$ week of pregnancy [12]. After the $17^{\text {th }}$ week, the size of the uterus makes laparoscopic TAC unattainable and laparotomic approach the only option for patients with $\mathrm{Cl}$ who cannot have TVC, after the first trimester of pregnancy [5].

The main advantage of trans-abdominal TAC is that the cervix has reached its full size and shape. That helps the surgeon to apply the optimal tension of the cerclage band at knotting in the cervix [8]. It seems that putting the cerclage in a non-distended uterus may not be effective some times; the cerclage band may loosen and this may lead to abortion or premature labor or the cerclage may be too tight for the cervix during pregnancy, which can result in tissue necrosis [13-19].

Before cerclage suture placement, clinicians should confirm the absence of chorioamnionitis, and the well-being of the fetus [chromosomal or congenital anomalies or fetal demise]. Cerclage placement is also contraindicated in cases with vaginal bleeding of unknown origin, as well as in premature membrane rupture.

TAC placement may have difficulties, mainly attributed to uterine manipulation, because of the advanced gestational age; excessive manipulation may cause uterine contractions and as a result, preterm labor or membrane rupture [5]. Inappropriate placement of the cerclage band in the area of the uterine artery branches may cause excessive bleeding, or even trauma to the ureters. This is the reason why TAC should be performed only by dedicated surgeons. Another reason which may potentially increase the morbidity of TAC, is that the patients need to undergo two operations: the first for placement of the cerclage and the second for the C-section.

However, despite the higher morbidity rates compared to TVC, TAC seems to have high neonatal survival rates. In the literature, the reported survival rates range between $60 \%$ and $96 \%$ [1315]. Song et al. [4] reported a high fetal survival rate equal to $96 \%$ and higher rates of delivery at $>34$ weeks of gestation [95\%] in TAC pregnancies; furthermore, the authors suggested that the successful outcomes in TAC cases may be attributed to the position of the cerclage band and the knot which are placed higher compared to TVC placement. Therefore, TAC technique may potentially prevent an inflammatory reaction, resulting in prolongation of the pregnancy.

Cervical length is another predictive factor of successful TAC [4]; the shorter the cervical length is at 20-24 weeks of pregnancy, the higher the possibility of preterm labor before 34 weeks, after TAC. In our case, despite the complete dilation of the cervix, TAC placement was successfully performed without further complications and the labor began at week 34 of gestation.

To the best of our knowledge, this is the first case in the medical literature of emergency TAC, which took place at the $22 \mathrm{nd}$ week of pregnancy. The fetal and maternal risks at this gestational age are extremely high. In our case, the uterus was carefully and gently elevated out of the abdominal cavity through a Pfannestiel incision, despite its size. Although this maneuver might predispose to uterine contractions or rupture of membranes, we believe, it is absolutely necessary as it gives the surgeon the opportunity to identify the area of the isthmus as well as the branches of the uterine artery and the avascular area between them. Pulling out and putting in again the 22-weeks uterus should be done by an extremely experienced surgeon, in order to avoid possible complications. Indeed, in our case, cerclage placement was performed by an experienced surgeon [S.F., 25 years of obstetrical experience].

We proceeded to emergency TAC placement, as it was the only option to prolong the pregnancy. Despite the complete cervical dilation in our patient, which, is considered an adverse prognostic factor for the success of the cerclage and for full term labor [ $>34^{\text {th }}$ week] [4], delivery began at 34+0 week.

\section{Conclusion}

To the best of our knowledge, this is the first case of an emergency TAC performed in gestation $>21$ weeks, our patient successfully completed her pregnancy, without any complication. Therefore, we believe, that TAC placement even in mid-term pregnancy, may be a safe procedure for both mother and fetus; however, it requires expertise and should be performed under strict criteria. 


\section{References}

1 Zaveri V, Aghajafari F, Amankwah K, Hannah M (2002) Abdominal versus vaginal cerclage after a failed transvaginal cerclage: A systematic review. Am J Obstet Gynecol 187: 868-872.

2 SOGC Clinical Practice Guidelines (2013) Cervical insufficiency and cervical cerclage. No.301.

3 Drakeley AJ, Roberts D, Alfirevic Z (2003) Cervical stitch (cerclage) for preventing pregnancy loss in women. Cochrane Database of Systematic Reviews.

4 Song JE, Lee KY, Son GH (2014) Prediction of outcome for trans abdominal cerclage in women with cervical insufficiency. Biomed Research International 2015: 1-5.

5 Joung EJ, Go EB, Kwack JY, Kwon YS (2016) Successful term delivery cases of trans-abdominal cervico isthmic cerclage performed at more than 18 weeks of gestation. Obstet Gynecol Sci 59(4): 319-322

6 Besonand RC, Durfee RB (1965) Trans abdominal cervico uterine cerclage during pregnancy for the treatment of cervical incompetency. Obstet Gynecol 25(2): 145-155.

7 Pereira RM, Zanatta A, Bianchi PH, Yadid IM, Motta EL, et al. (2009) Successful interval laparoscopic trans abdominal cervico isthmic cerclage preceding twin gestation: a case report. J Minim Invasive Gynecol 16: 634-638.

8 Witt MU, Joy SD, Clark J, Herring A, Bowes W, et al. (2009) Cervico isthmic Cerclage: Trans abdominal versus Trans vaginal Approach. Am J Obstet Gynecol 201(1): 105.e1-4

9 Althuisius SM, Dekker GA, Hummel P, Geijn HP van (2003) Cervical incompetence prevention randomized cerclage trial: emergency cerclage with bed rest versus bed rest alone. Am J Obstet Gynecol 189: $907-910$

10 Daskalakis G, Papantoniou N, Mesogitis S, Antsaklis A (2006) Management of cervical insufficiency and bulging fetal membranes. Obstet Gynecol 107: 221-226.

11 Stupin JH, David M, Siedentopf JP, Dudenhausen JW (2008) Emergency cerclage versus bed rest for amniotic sac prolapse before 27 gestation weeks. A retrospective, comparative study of 161 women. Eur J Obtet Gynecol Reprod Biol 139: 32-37.

12 Schorr SJ, Morales WJ (1996) Obstetric management of incompetent cervix and bulging fetal membranes. J Reprod Med 41: 235-238.

13 Olatunbosun OA, Al-Nuaim L, Turnell RW (1995) Emergency cerclage compared with bed rest for advanced cervical dilatation in pregnancy. Int Surg 80: 170-174.

14 Shirodkar V (1955) A new method of operative treatment of habitual abortions in the second trimester of pregnancy. Antiseptic 52: 299-300.

15 McDonald (1957) Suture of the cervix for inevitable miscarriage. J Obstet Gynaecol Br Emp 64: 346-353.

16 Turnquest MA, Britton KA, Brown HL (1999) Outcome of patients undergoing trans abdominal cerclage: a descriptive study. J Mat-Fet Neonat Med 8(5): 225-227.

17 Gebruers M, Jacquemyn Y, Cornette J (2013) Laparoscopic trans abdominal cerclage. SurgSci 4: 231-235

18 Lotgering FK, Gaugler-Senden IPM, Lotgering SF, Wallenburg HCS (2006) Outcome after trans abdominal cervico isthmic cerclage. Obstet Gyne 107(4): 779-784.

19 Fick AL, Caughey AB, Parer JT (2007) Trans abdominal cerclage: Can we predict who fails? J Matel Fet Neonal Med 20(1): 63-67. 